\title{
Sensitivity to Immune Checkpoint Blockade in Advanced Non-Small Cell Lung Cancer Patients with EGFR Exon 20 Insertion Mutations
}

\author{
Giulio Metro ${ }^{1, *}$, Sara Baglivo ${ }^{1}(0)$, Guido Bellezza ${ }^{2}$, Martina Mandarano ${ }^{2}\left({ }^{\circ}\right.$, Alessio Gili ${ }^{3}{ }^{\circ}$, Giovanni Marchetti $^{4}$, \\ Marco Toraldo ${ }^{5}$, Carmen Molica ${ }^{1}$, Maria Sole Reda ${ }^{1}$, Francesca Romana Tofanetti ${ }^{1}$, Annamaria Siggillino ${ }^{1}$, \\ Enrico Prosperi ${ }^{6}$, Antonella Giglietti ${ }^{7}$, Bruna Di Girolamo ${ }^{8}$, Miriam Garaffa ${ }^{1}$, Francesca Marasciulo ${ }^{1}$, \\ Vincenzo Minotti ${ }^{1}$, Marco Gunnellini ${ }^{9}$, Annalisa Guida ${ }^{10}$, Monica Sassi ${ }^{7}$, Angelo Sidoni ${ }^{2}$, Fausto Roila ${ }^{11}$ and \\ Vienna Ludovini ${ }^{1}$ (I)
}

check for updates

Citation: Metro, G.; Baglivo, S.; Bellezza, G.; Mandarano, M.; Gili, A.; Marchetti, G.; Toraldo, M.; Molica, C.; Reda, M.S.; Tofanetti, F.R.; et al. Sensitivity to Immune Checkpoint Blockade in Advanced Non-Small Cell Lung Cancer Patients with EGFR Exon 20 Insertion Mutations. Genes 2021, 12, 679. https://doi.org/ $10.3390 /$ genes 12050679

Academic Editor: Paolo Cinelli

Received: 23 March 2021

Accepted: 26 April 2021

Published: 30 April 2021

Publisher's Note: MDPI stays neutral with regard to jurisdictional claims in published maps and institutional affiliations.

Copyright: (c) 2021 by the authors. Licensee MDPI, Basel, Switzerland. This article is an open access article distributed under the terms and conditions of the Creative Commons Attribution (CC BY) license (https:/ / creativecommons.org/licenses/by/ $4.0 /)$.
1 Medical Oncology, Santa Maria della Misericordia Hospital, via Dottori 1, 06156 Perugia, Italy; sara.baglivo@ospedale.perugia.it (S.B.); carmen.molica@ospedale.perugia.it (C.M.); mariasole.reda@ospedale.perugia.it (M.S.R.); francesca.tofanetti@ospedale.perugia.it (F.R.T.); annamaria.siggillino@ospedale.perugia.it (A.S.); miriam.garaffa@libero.it (M.G.); marasciulofrancesca@libero.it (F.M.); vincenzo.minotti@ospedale.perugia.it (V.M.); vienna.ludovini@ospedale.perugia.it (V.L.)

2 Section of Anatomic Pathology and Histology, Department of Medicine and Surgery, University of Perugia, Piazza Lucio Severi 1, 06132 Perugia, Italy; guido.bellezza@unipg.it (G.B.); martina.mandarano@unipg.it (M.M.); angelo.sidoni@unipg.it (A.S.)

3 Public Health Section, Department of Experimental Medicine, University of Perugia, Piazza Lucio Severi 1, 06132 Perugia, Italy; alessio.gili@gmail.com

4 Division of Pathology, Santa Maria Hospital, via Tristano di Joannuccio 1, 05100 Terni, Italy; g.marchetti@aospterni.it

5 Division of Pathology, San Giovanni Battista Hospital, via Massimo Arcamone, 06034 Foligno, Italy; marco.toraldo@uslumbria2.it

6 Division of Pathology, Città di Castello Hospital, Via Luigi Angelini 10, 06012 Città di Castello, Italy; enrico.prosperi@uslumbria1.it

7 Hematology and Oncology Unit, San Giovanni Battista Hospital, via Massimo Arcamone, 06034 Foligno, Italy; antonella.giglietti@uslumbria2.it (A.G.); monica.sassi@uslumbria2.it (M.S.)

8 Oncologic Day Hospital, Santa Maria della Stella Hospital, Località Ciconia, 05018 Orvieto, Italy; bruna.digirolamo@uslumbria2.it

9 Medical Oncology, Gubbio and Gualdo Tadino Hospital, Largo Unità d'Italia, 06024 Branca, Italy; marco.gunnellini@uslumbria1.it

10 Medical Oncology, Santa Maria Hospital, via Tristano di Joannuccio 1, 05100 Terni, Italy; a.guida@aospterni.it

11 Medical Oncology, Santa Maria della Misericordia Hospital, University of Perugia, Piazza Lucio Severi 1, 06132 Perugia, Italy; fausto.roila@unipg.it

* Correspondence: giulio.metro@ospedale.perugia.it; Tel.: +39-0755783695; Fax: +39-075-5784184

Abstract: Besides platinum-based chemotherapy, no established treatment option exists for advanced non-small-cell lung cancer (NSCLC) patients with EGFR exon 20 (Ex20ins) insertion mutations. We sought to determine the clinical outcome of patients with this EGFR mutation subtype in the immunotherapy era. Thirty NSCLCs with EGFR Ex20ins mutations were identified, of whom 15 had received immune checkpoint blockade (ICB) treatment as monotherapy $(\mathrm{N}=12)$, in combination with chemotherapy $(\mathrm{N}=2)$ or with another immunotherapeutic agent $(\mathrm{N}=1)$. The response rate was observed in 1 out of 15 patients (6.7\%), median progression-free survival (PFS) was 2.0 months and median overall survival (OS) was 5.3 months. A trend towards an inferior outcome in terms of PFS and OS was observed for patients receiving ICB treatment in the first versus second line setting (PFS: 1.6 months versus 2.7 months, respectively, $p=0.16-\mathrm{OS}: 2.0$ months versus 8.1 months, respectively, $p=0.09$ ). Median OS from the time of diagnosis of advanced disease was shorter for patients treated with ICB versus those who did not receive immunotherapy (12.9 months versus 25.2 months, respectively, $p=0.08)$, which difference remained associated with a worse survival outcome at multivariate analysis $(p=0.04)$. Treatment with ICB is poorly effective in NSCLCs with EGFR Ex20ins mutations, especially when given in the first-line setting. This information is crucial in order to select the optimal treatment strategy for patients with this subtype of EGFR mutation. 
Keywords: EGFR exon 20 insertion mutations (Ex20ins); immune checkpoint blockade (ICB); immunotherapy; non-small-cell lung cancer (NSCLC); PD-L1

\section{Introduction}

Two major treatment paradigms have been recently established for the management of advanced non-small-cell lung cancer (NSCLC), either targeted therapies based on the inhibition of aberrant oncogenic drivers or immunotherapy based on the blocking of immunosuppressive checkpoints [1]. Targeting the epidermal growth factor receptor (EGFR) mutation with an EGFR-tyrosine kinase inhibitor (-TKI) is a highly effective treatment strategy for patients with certain types of EGFR mutation, such as those located at exon 19 (deletions) or 21 (L858R point mutations), which comprise approximately $90 \%$ of all EGFR mutations [2]. Similarly, some uncommon EGFR mutations may still respond to treatment with an EGFR-TKI (i.e., G719, L861Q and S861Q), although to a less extent compared with common EGFR exon 19 deletion/L858R point mutations. By contrast, NSCLCs with EGFR exon 20 insertion mutations (Ex20ins), which constitute a heterogeneous group of genetic in-frame insertions in exon 20 of EGFR, are generally insensitive (with very few exceptions) to EGFR-TKI targeted treatment [3]. Therefore, platinum-based chemotherapy is to be considered the standard first-line treatment for NSCLCs with EGFR Ex20ins mutations; however, this results in a dismal prognosis as no other validated options are available at the time of disease progression.

Treatment with immune checkpoint blockade (ICB) with programmed cell death-1 (PD-1)/programmed cell death ligand-1 (PD-L1)/cytotoxic T-lymphocyte antigen 4 (CTLA4) monoclonal antibodies has led to unprecedented durable clinical benefit for NSCLC, but response rates are low for patients with oncogenic drivers such as EGFR mutations $[4,5]$. However, the impact of uncommon subtypes of EGFR mutations on the efficacy of treatment with ICB has been less explored. While uncommon non-Ex20ins EGFR mutations seem to experience some benefit from ICB treatment, little is known about the correlation existing between NSCLCs with EGFR Ex20ins mutations and response to immunotherapy [6]. In more detail, it is not known whether NSCLCs harboring EGFR Ex20ins mutations are suitable for treatment with ICB either in the initial 'naïve' setting or resistant clinical scenario after platinum-based chemotherapy.

In this multicenter study we aimed to evaluate the clinicopathological and molecular characteristics of advanced NSCLC patients with EGFR Ex20ins in order to associate them with clinical outcome according to ICB treatment. The final intent of this analysis was to assess whether immunotherapy could represent a valuable therapeutic strategy for this group of patients.

\section{Materials and Methods}

\subsection{Study Population}

Patients with advanced (either locally advanced inoperable or metastatic) NSCLC and EGFR Ex20ins mutations who had received at least one anti-cancer therapy were identified from a prospectively maintained institutional database. Patients were enrolled at 5 different Institutions (Santa Maria della Misericordia Hospital in Perugia, San Giovanni Battista Hospital in Foligno, Santa Maria Hospital in Terni, Santa Maria della Stella Hospital in Orvieto and Gubbio and Gualdo Tadino Hospital in Branca). The medical records of these patients were retrospectively collected, and only patients with clinically available data were considered to be eligible. All patients whose EGFR Ex20ins mutation were initially detected by direct sequencing were retrospectively subjected to confirmation by next generation sequencing (NGS) (see below), which was implemented at Perugia Hospital since June 2016. 


\subsection{NGS Analysis}

NGS analysis was performed in the Molecular Biology Laboratory of Medical Oncology Division at S. Maria della Misericordia Hospital in Perugia, Italy. For DNA extraction, $2-5$ sections of $10 \mu \mathrm{m}$-thick formalin-fixed paraffin-embedded (FFPE) tissues were prepared. One slide at the beginning of each serial section was stained with hematoxylin-eosin and histopathologically examined to determine the tumor cell content. Only samples with a sufficient tumor cell content were included in the study. After macrodissection of the tumor area, DNA was isolated using a QIAamp DNA FFPE Tissue kit (Qiagen GmbH, Hilden, Germany) on QiaCube robotic workstation according to the manufacturer's instructions. DNA concentrations were determined with the Qubit DNA HS Assay Kit and Qubit 3.0 Fluorometer (Thermo Fisher Scientific, Carlsbad, CA, USA). Starting from 10 ng of DNA, libraries were prepared manually using the Ion AmpliSeq ${ }^{\mathrm{TM}}$ Library kit 2.0 and Ion AmpliSeq $^{\mathrm{TM}}$ Colon and Lung Cancer Research Panel v2 (Thermo Fisher Scientific, Carlsbad, CA, USA) to amplify hotspots and targeted and regions of 22 genes (KRAS, EGFR, BRAF, PIK3CA, AKT1, ERBB2, PTEN, NRAS, STK11, MAP2K1, ALK, DDR2, CTNNB1, MET, TP53, $S M A D 4, F B X 7, F G F R 3$, NOTCH1, ERBB4, FGFR1 and FGFR2). Equalized purified libraries were combined and diluted to obtain a 16-Ion Express Barcode 30pM single pool. Template preparation and enrichment were performed using the Ion Chef ${ }^{\mathrm{TM}}$ system and Ion $510^{\mathrm{TM}}$, Ion $520^{\mathrm{TM}}$ and Ion $530^{\mathrm{TM}}$ Kit-Chef (Thermo Fisher Scientific, Carlsbad, CA, USA). Sequencing was performed on S5 System using an Ion 510 Chip and Ion S5 ${ }^{\mathrm{TM}}$ sequencing kit. Signal processing and base calling ware carried out basing analysis on the default base-caller parameters of Torrent Suite (v.5.12). Variants with a quality $<30$ were filtered out. NGS data analysis was performed using Ion Reporter. The limit of detection (LOD) for single nucleotide variants (SNVs), insertions / deletions and splice site mutations was $\geq 3 \%$ mutant allele frequency (MAF) with a minimum depth of $500 \times$. The frequency of each mutant allele was recorded. Amplicon reads were reviewed with an Integrative Genomics Viewer (IGV) allowing fir visual inspection of the coverage of the interested regions. Alignment and variant calling were performed using human reference genome 19 (hg19).

\subsection{Tumor PD-L1 Analysis}

Tumor PD-L1 analysis was performed in the Section of Anatomic Pathology and Histology of Perugia University, Italy. Immunohistochemistry (IHC) for PD-L1 was performed using the PD-L1 22C3 pharmDx kit (Dako North America Inc., Carpinteria, CA, USA) on the Dako Autostainer Link 48, according to the manufacturers' instructions. Unstained tissue section $4-\mu \mathrm{m}$ thick were prepared from the most representative area of each case. At least 100 viable tumor cells were required for a valid interpretation of PD-L1 staining. Slides were counterstained with Mayer's hematoxylin. Results were evaluated with known positive and negative tissue controls. The percentage of PD-L1 expression on invasive tumor cells was calculated as the number of viable invasive carcinoma cells showing membranous staining of any intensity divided by the total number of viable invasive carcinoma cells.

\subsection{Statistical Analysis}

The sample size was estimated according to the expected enrollment of the participating centers and to the purely descriptive intent of the study itself. The primary endpoints were to assess the clinicopathological and molecular characteristics of advanced NSCLC patients with EGFR Ex20ins mutations and describe their clinical outcome according to ICB treatment in terms of response rate (RR), progression-free survival (PFS) and overall survival (OS). The secondary endpoints were to evaluate the overall clinical outcome of patients with EGFR Ex20ins mutations according to whether they had received ICB treatment, as well as to perform univariate and multivariate analyses for OS in all patients in order to assess whether any baseline clinical factors would affect clinical outcome. Responses were assessed with RECIST (version 1.1) criteria [7]. Progression-free survival was the time elapsing between the start of ICB treatment and disease progression at any site or death in the absence of documented disease progression, while OS was calculated from 
the start of ICB treatment (for the primary endpoint) or from the diagnosis of advanced disease (for the secondary endpoints) until death for any cause. Descriptive statistics were calculated including frequencies, percentages, frequency tables for categorical variables, median and means \pm standard deviation for quantitative variables. Categorical variables were evaluated by Chi-square or Fisher's exact test when appropriate. The Kaplan-Meier method was used to analyze OS, PFS and to estimate medians of survival with two-sided $95 \%$ confidence intervals (CI). Survival curves were compared using the log-rank test. Cox regression model (univariate and multivariate) was applied to estimate the Hazard Ratio (HR) and $95 \% \mathrm{CI}$ and to identify prognostic factors independently associated with survival times. Stepwise backward-selection was used for elimination of variables from the regression model to find a reduced model that best explains the data. All the covariates found to be statistically significant in the univariate approach were included in the multivariate mode. A $p$-value of less than 0.05 was considered to be statistically significant. Statistical analyses were performed with STATA v. 16.1 (StataCorp LP, College Station, TX, USA). The procedures followed were in accordance with rules of the local Ethics Committee and the Declaration of Helsinki. The study was approved by the Ethical Committee CEAS Umbria. (prot. No. 21274).

\section{Results}

\subsection{Patients}

From August 2008 until October 2020, 30 patients harboring an EGFR Ex20ins mutation were eligible for inclusion in the study. Figure $\mathrm{S} 1$ shows the study flowchart. The A767_V769dup and H773_V774insNPH variants were the most frequently identified variants, comprising $34 \%$ of EGFR Ex20ins cases ( $\mathrm{N}=517 \%$ and $\mathrm{N}=517 \%$, respectively). EGFR Ex20ins patients had at least one concomitant mutation in 17 cases $(56.7 \%)$, including TP53 $(\mathrm{N}=14,46.7 \%)$ and SMAD4 $(\mathrm{N}=2,6.7 \%)$. Figure 1A shows the distribution of EGFR Ex20in variants, while Figure $1 \mathrm{~B}$ indicates the distribution of EGFR Ex20ins mutations along with concomitant genetic alterations found at NGS. Table 1 lists the patients' characteristics and classifies them according to whether they had received immunotherapy $(\mathrm{N}=15)$. In all cases the histological subtype consisted of adenocarcinoma. Overall, median age was 59 years, slightly more than half of patients were never smokers, and approximately one third of patients had a PD-L1 $\geq 1 \%$. Apparently, there were no statistically significant differences between patients with EGFR Ex20ins mutations who received ICB treatment versus those who did not, except for the presence of a higher number of patients with PD-L1 $\geq 1 \%$ in the immunotherapy group. Further details on the type of treatments received besides immunotherapy for each group of patients are described in Table S1. Table 2 shows some other key characteristics of the 15 patients who were treated with ICB treatment. Eighty percent of patients (12/15) were treated with an anti-PD1 or anti-PD-L1 agent as monotherapy, while immunotherapy was administered as first line treatment in $40 \%$ of patients $(6 / 15)$. Of them, 4 patients received pembrolizumab as monotherapy because of PD-L1 $\geq 50 \%$, while 2 patients who had a PD-L1 of $25 \%$ and $<1 \%$ were treated with platinum-based chemotherapy in combination with pembrolizumab. 
A
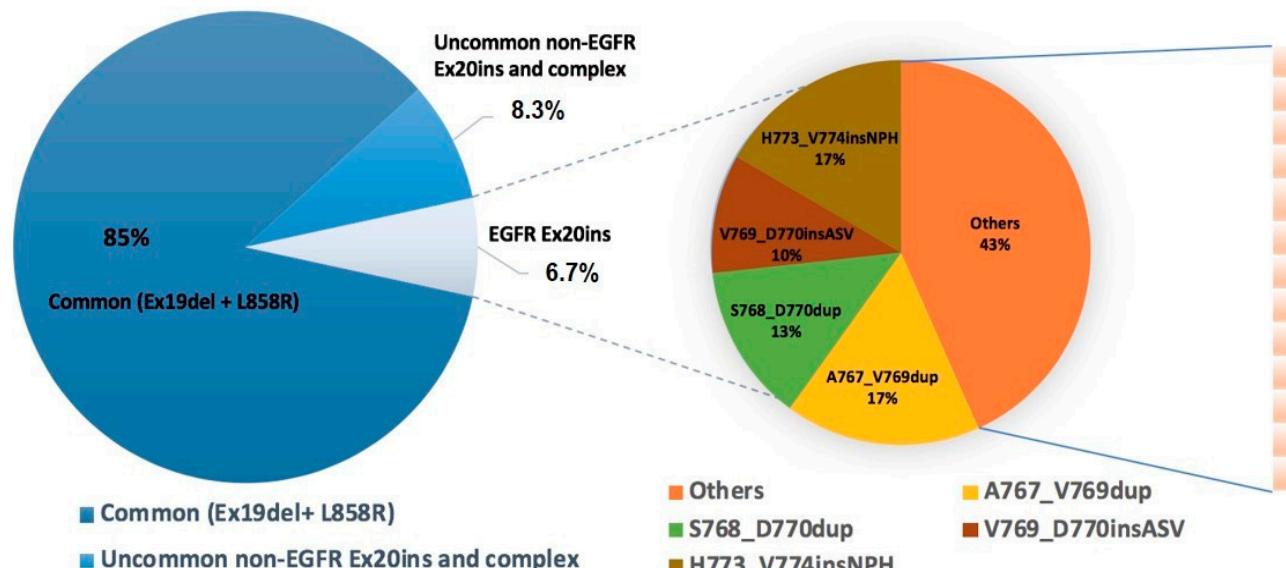

A766_V768insMAS

S768_V769insMDS

p.V769_D770insGSV

p.V769_D770insGFV

D770_N771insNPY+ H773Y

N771_P772insV

N771_P772delinsSVDNT

N771delinsHH

H773_V774insAPH

H773_V774insH

His773_V774insAH

H773delinsYNPY

p.H773delinsPNPY

- Common (Ex19del+ L858R)

ancommon non-EGFR Ex20ins and complex

-H773_V774insNPH

B

$$
\text { EGFR Ex20ins }
$$

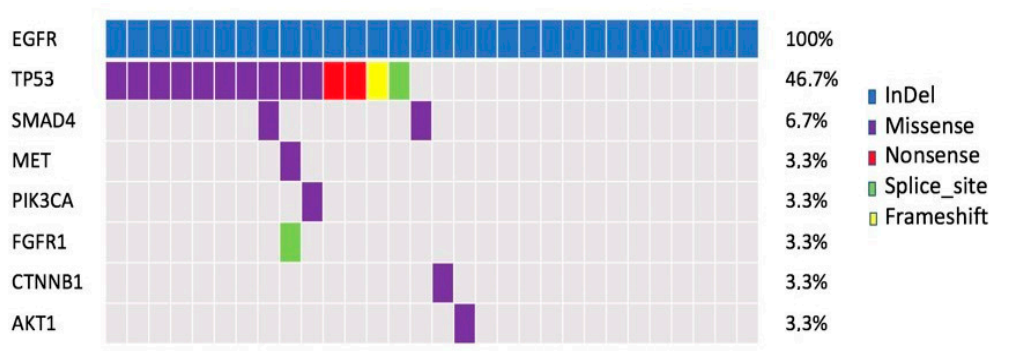

C

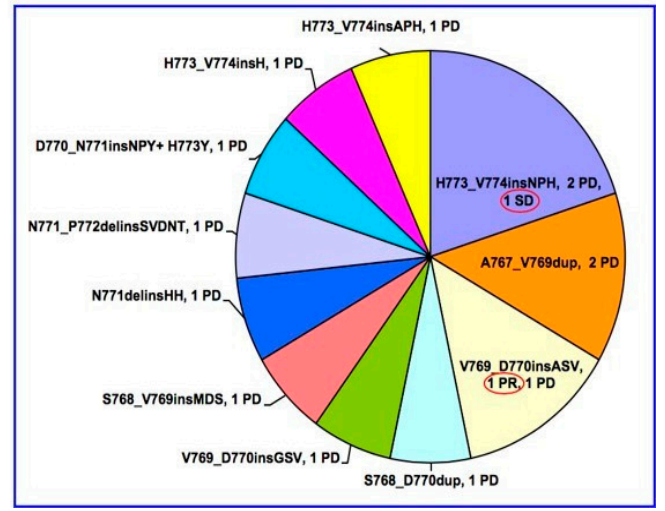

Figure 1. Distribution of types of EGFR mutations and EGFR Ex20ins mutations in the study cohort $(\mathrm{N}=445)(\mathrm{A})$; the distribution of EGFR Ex20ins mutations and concomitant mutations stratified according to NGS sequencing and type of alteration. Each column corresponds to one of the 30 patients (B); the distribution of EGFR Ex20ins variants and the corresponding response to immune checkpoint blockade. Abbreviations: PR, Partial Response; SD, Stable Disease; PD, Progressive Disease (C).

Table 1. Characteristics of patients.

\begin{tabular}{|c|c|c|c|c|}
\hline Characteristic & $\begin{array}{c}\text { Total } \\
\mathrm{N}=30(\%)\end{array}$ & $\begin{array}{l}\text { Immunotherapy } \\
\quad \mathrm{N}=15(\%)\end{array}$ & $\begin{array}{l}\text { No Immunotherapy } \\
\qquad N=15(\%)\end{array}$ & $p$-Value \\
\hline Median Age, Years (Range) & $59(33-88)$ & $58(45-79)$ & $64(33-88)$ & 0.15 \\
\hline \multicolumn{5}{|l|}{ Gender } \\
\hline Male & $13(43.3)$ & $6(40.0)$ & $7(46.7)$ & 0.71 \\
\hline Female & $17(56.7)$ & $9(60.0)$ & $8(53.3)$ & \\
\hline \multicolumn{5}{|l|}{ Smoking history } \\
\hline Ever & $13(43.3)$ & $6(40.0)$ & $7(46.7)$ & 0.71 \\
\hline Never & $17(56.7)$ & $9(60.0)$ & $8(53.3)$ & \\
\hline \multicolumn{5}{|l|}{ Stage IV at first diagnosis } \\
\hline Yes & $26(86.7)$ & $14(93.3)$ & $12(80.0)$ & 0.28 \\
\hline No & $4(13.3)$ & $1(6.7)$ & $3(20.0)$ & \\
\hline \multicolumn{5}{|l|}{ PS at diagnosis of advanced disease } \\
\hline $0-1$ & $24(80.0)$ & $11(73.3)$ & $13(86.7)$ & 0.36 \\
\hline 2 & $6(20.0)$ & $4(26.7)$ & $2(13.3)$ & \\
\hline \multicolumn{5}{|l|}{ Lines of treatment } \\
\hline$\leq 2$ & $20(66.7)$ & $9(60.0)$ & $11(73.3)$ & 0.44 \\
\hline$>3$ & $10(33.3)$ & $6(40.0)$ & $4(26.7)$ & \\
\hline
\end{tabular}


Table 1. Cont

\begin{tabular}{|c|c|c|c|c|}
\hline Characteristic & $\begin{array}{c}\text { Total } \\
\mathrm{N}=30(\%)\end{array}$ & $\begin{array}{l}\text { Immunotherapy } \\
\qquad N=15(\%)\end{array}$ & $\begin{array}{l}\text { No Immunotherapy } \\
\qquad N=15(\%)\end{array}$ & $p$-Value \\
\hline \multicolumn{5}{|c|}{ Any platinum-based chemotherapy treatment } \\
\hline Yes & $23(76.7)$ & $11 *(73.3)$ & $12(80.0)$ & 0.51 \\
\hline No & $7(23.3)$ & $4(26.7)$ & $3(20.0)$ & \\
\hline \multicolumn{5}{|c|}{ Any EGFR-TKI treatment } \\
\hline Yes & $11(36.7)$ & $4(26.7)$ & $7(46.7)$ & 0.26 \\
\hline No & $19(63.3)$ & $11(73.3)$ & $8(53.3)$ & \\
\hline \multicolumn{5}{|l|}{ PD-L1 status } \\
\hline$\geq 1 \%$ & $10(33.3)$ & $9^{* *}(60.0)$ & $1^{* * *}(6.7)$ & \\
\hline$<1 \%$ & $15(50.0)$ & $5(33.3)$ & $10(66.7)$ & 0.007 \\
\hline Unknown & $5(16.7)$ & $1(6.7)$ & $4(26.6)$ & \\
\hline \multicolumn{5}{|l|}{ TP53 mutation } \\
\hline Present & $14(46.7)$ & $8(53.3)$ & $6(40.0)$ & \\
\hline Absent & $13(43.3)$ & $7(46.7)$ & $6(40.0)$ & 0.19 \\
\hline Unknown & $3(10.0)$ & 0 & $3(20.0)$ & \\
\hline
\end{tabular}

EGFR-TKI, epidermal growth factor receptor-tyrosine kinase inhibitor; N, number; PD-L1, programmed death ligand-1; PS, performance status; ${ }^{*} 2$ patients received platinum-based chemotherapy + pembrolizumab; ${ }^{* *} 1$ patient $80 \%, 3$ patients $60 \%, 2$ patient $40 \%, 1$ patient $35 \%$, 1 patient $25 \%, 1$ patient $10 \%$; ${ }^{* * *} 1$ patient $15 \%$.

Table 2. Key characteristics of patients treated with immune checkpoint blockade.

\begin{tabular}{|c|c|}
\hline Characteristic & $\begin{array}{c}\text { Total } \\
\mathrm{N}=15(\%)\end{array}$ \\
\hline Treatment & \\
\hline Anti-PD1 * & $10(66.7)$ \\
\hline Anti-PD-L1 ** & $2(13.3)$ \\
\hline Anti-PD-1 + anti-CTLA4 ${ }^{* * *}$ & $1(6.7)$ \\
\hline Platinum-based chemotherapy + anti-PD1 ${ }^{+}$ & $2(13.3)$ \\
\hline \multicolumn{2}{|l|}{ Line of immune checkpoint blockade administration } \\
\hline 1 st & $6(40.0)$ \\
\hline$\geq 2$ nd & $9(60.0)$ \\
\hline \multicolumn{2}{|l|}{ PS prior to immune checkpoint blockade } \\
\hline $0-1$ & $11(73.3)$ \\
\hline 2 & $4(26.7)$ \\
\hline
\end{tabular}

PD-1, programmed death-1; PD-L1, programmed death ligand-1; PS, performance status; ${ }^{*} 6$ patients pembrolizumab and 4 patients nivolumab; ${ }^{* *} 2$ patients atezolizumab; ${ }^{* * *}$ Nivolumab + ipilimumab; ${ }^{\dagger} 2$ patients platinum-pemetrexed + pembrolizumab.

\subsection{Activity of Treatment with Immune Checkpoint Blockade}

Table 3 shows the activity of treatment with the immune checkpoint blockade. Only one patient (6.7\%) experienced a partial response, with 11 patients $(73.3 \%)$ showing progressive disease as their best response. Figure $1 \mathrm{C}$ shows the distribution of response according to the type of EGFR Ex20ins variant. The only patient who responded to ICB treatment was found to have a V769_D770insAV. However, the same mutation was found in a patient who experienced PD as best response. Of the 6 patients who received treatment with immune ICB as first line therapy, 4 patients were evaluable for response. Of them there were 3 patients treated with pembrolizumab as monotherapy and 1 patient treated with platinum-based chemotherapy in combination with pembrolizumab, all of whom underwent a progressive disease. The other 2 patients treated in first line with ICB (1 with pembrolizumab as monotherapy and 1 with platinum-based chemotherapy in combination with pembrolizumab) were not evaluable for response because of a rapid deterioration of clinical conditions after the administration of only one cycle of treatment, with subsequent death in the absence of radiological disease reassessment. Only 2 of the 6 patients treated in the first line setting (both of whom were treated with pembrolizumab as monotherapy) 
were able to receive a second line treatment consisting of platinum-based chemotherapy in one case and the EGFR-TKI inhibitor poziotinib in another case. At a median follow-up of 4.6 months (range 0.7-21.6), median PFS was 2.0 months (95\% CI 0.6-2.7), while median OS was 5.3 months (95\% CI 1.8-12.5) (Figure 2A,D). Patients treated in the first line setting experienced a worse outcome in terms of both PFS and OS; however, they did not reach statistical significance likely because of the low number of patients being compared (PFS: 1.6 months for first line versus 2.7 months for second line, $p=0.16$-OS: 2.0 months for first line versus 8.1 months for second line, $p=0.09$ ) (Figure 2B,E). By contrast, no apparent difference was noted for PFS and OS by PD-L1 status (PFS: 2.7 months for PD-L1 $<1 \%$ versus 2.0 months for PD-L1 $\geq 1 \%, p=0.39-\mathrm{OS}$ : 12.5 months for PD-L1 $<1 \%$ versus 4.2 months for PD-L1 $\geq 1 \%, p=0.58$ ) (Figure $2 \mathrm{C}, \mathrm{F}$ ).

Table 3. Response to treatment with immune checkpoint blockade.

\begin{tabular}{cc}
\hline Best Response & Total \\
& $\mathbf{N}=\mathbf{1 5}(\mathbf{\%})$ \\
\hline Partial response & $1(6.7)$ \\
Stable disease & $1(6.7)$ \\
Progressive disease & $11(73.3)$ \\
Not evaluable & $2 *(13.3)$ \\
\hline *
\end{tabular}
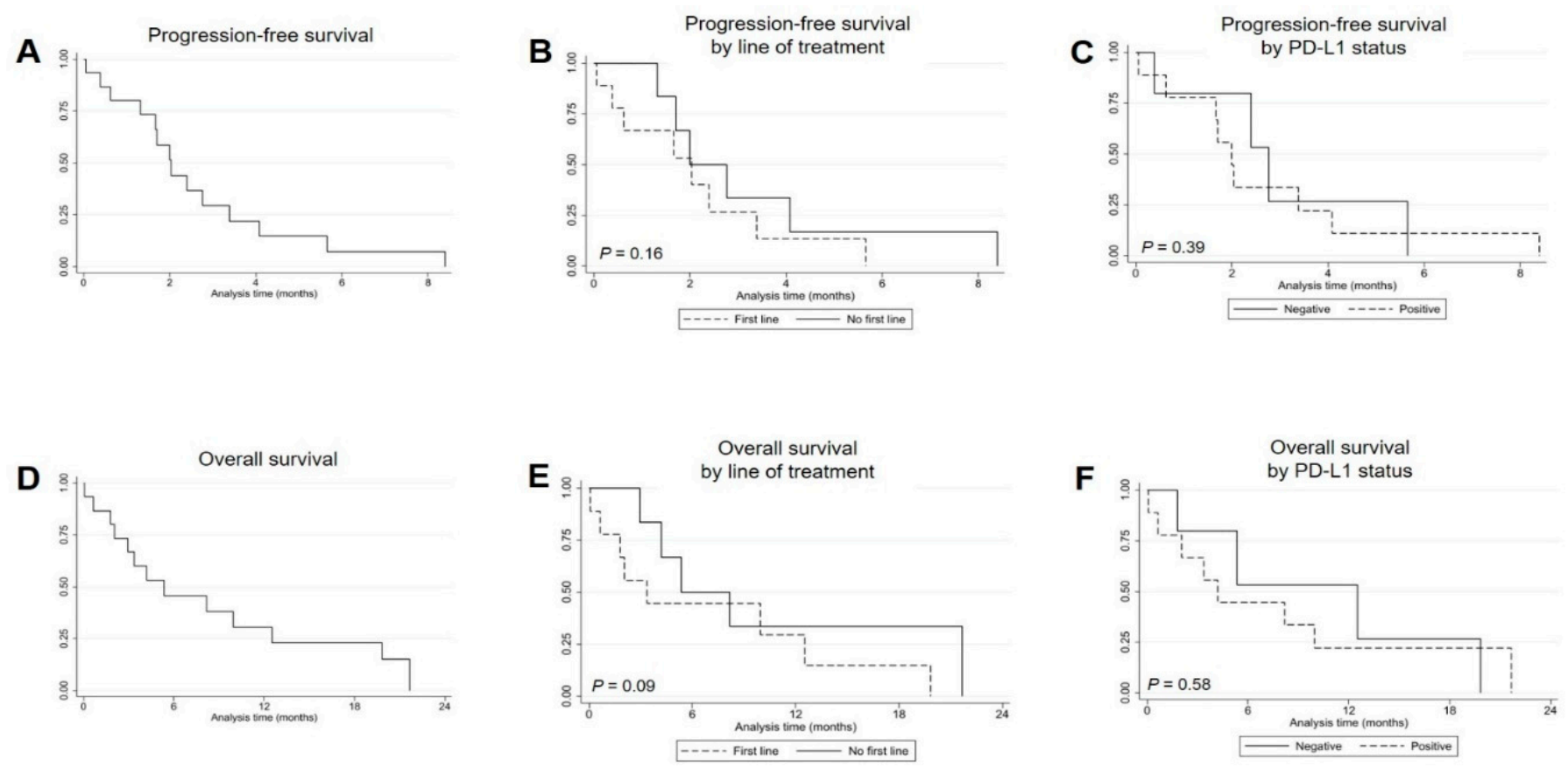

Figure 2. Kaplan-Meier curves of progression-free survival in all patients treated with immune checkpoint blockade (A) and by line of treatment (B) or PD-L1 status (C); Kaplan-Meier curves of overall survival in all patients treated with immune checkpoint blockade (D) and by line of treatment (E) or PD-L1 status (F).

\subsection{Overall Survival of All Patients and by Treatment with ICB}

We went on to evaluate the OS from the time of diagnosis of advanced disease for all patients with EGFR Ex20ins mutations and according to the type of treatment received. At a median follow-up of 13.2 months (range 1.4-40.4), the median OS for all patients was 17.2 months (Figure 3A). When patients were classified by whether they had received treatment with ICB, median OS was shorter, at the limit of significance, for patients treated with immunotherapy versus those who did not receive ICB treatment, being 12.9 months (95\% CI 13.3-35.0) versus 25.2 months (95\% CI 3.2-28.2) ( $p=0.08)$, respectively (Figure 3B). 
At multivariate analysis for OS, ICB treatment remained significantly associated with shorter OS $(p=0.04)$, along with other parameters such as performance status of 2 and having received a number of treatment lines $\leq 2$ (Table 4 ).
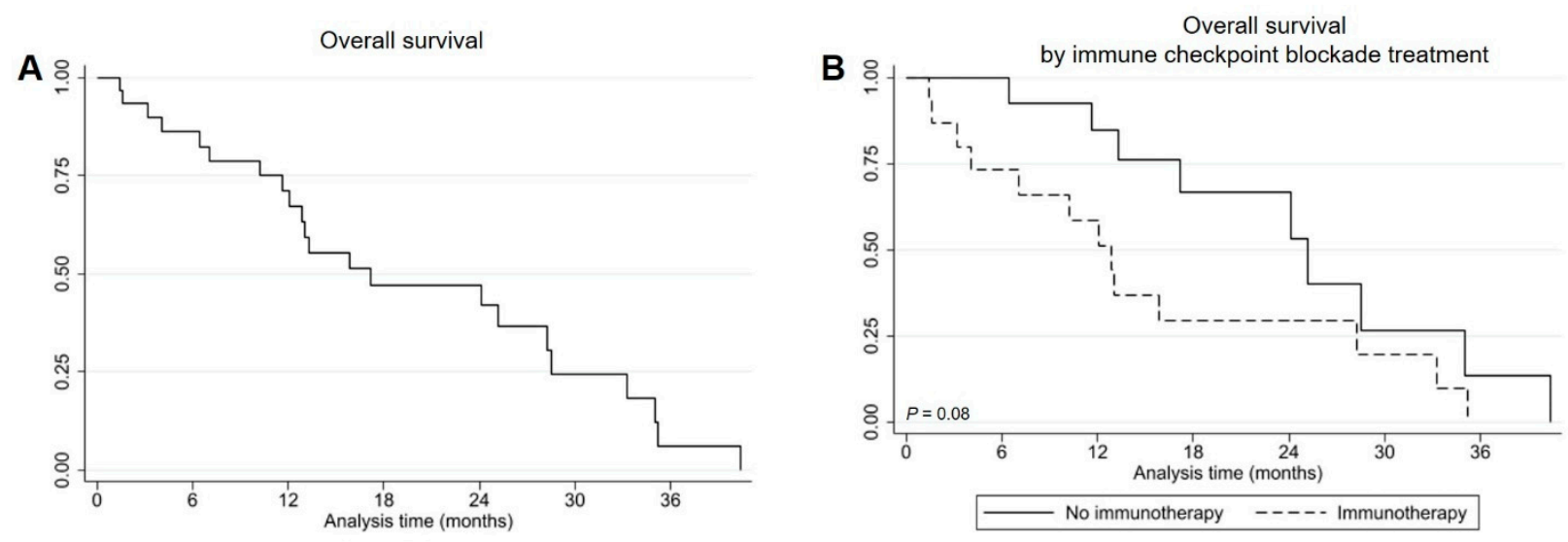

Figure 3. Kaplan-Meier curves of overall survival in all patients regardless of treatment with immune checkpoint blockade (A) and by immune checkpoint blockade treatment (B).

Table 4. Univariate and multivariate analyses of prognostic factors for overall survival.

\begin{tabular}{|c|c|c|c|c|}
\hline \multirow{2}{*}{ Factor } & \multicolumn{2}{|c|}{ Univariate Analysis } & \multicolumn{2}{|c|}{ Multivariate Analysis } \\
\hline & HR $(95 \%$ CI) & $p$-Value & HR $(95 \%$ CI) & $p$-Value \\
\hline Age, $\geq 65$ y vs. $<65$ y & $1.09(0.43-2.81$ & 0.85 & - & - \\
\hline Gender, male vs. female & $1.20(0.50-2.88)$ & 0.67 & - & - \\
\hline Smoking history, never vs. ever & $1.16(0.49-2.75)$ & 0.74 & - & - \\
\hline PS at diagnosis of advanced disease, 2 vs. $0-1$ & $4.79(1.44-15.82)$ & 0.01 & $8.64(2.01-35.6)$ & 0.003 \\
\hline Lines of treatment, $\leq 2$ vs. $>3$ & $2.87(1.01-8.14)$ & 0.05 & $4.05(1.30-12.63)$ & 0.02 \\
\hline ICB treatment, yes vs. no & $2.14(0.88-5.20)$ & 0.08 & $2.88(1.12-7.41)$ & 0.03 \\
\hline PD-L1, $\geq 1 \%$ vs. $<1 \%$ & $1.69(0.66-4.32)$ & 0.27 & - & - \\
\hline TP53, present vs. absent & $1.33(0.52-3.40)$ & 0.55 & - & - \\
\hline
\end{tabular}

ICB, immune checkpoint blockade; PD-L1, programmed death ligand-1; PS, performance status.

\section{Discussion}

In this multicenter study we evaluated the effects of ICB treatment on the outcome of advanced NSCLC patients with EGFR Ex20ins mutations and found very poor clinical results of immunotherapy. Response rate was $6.7 \%(1 / 15)$, while PD was observed in $73.3 \%$ $(11 / 15)$ of patients (Table 3). Additionally, median PFS was 2.0 months, and median OS was 5.3 months (Figure 2A,D). These very modest results in terms of clinical outcomes suggest that ICB treatment is poorly effective in patients with EGFR Ex20ins mutations. Data available in the literature for NSCLCs with EGFR Ex20ins mutations treated with ICB are very scant and often refer to a limited number of patients. Chen et al. found a RR of $22.2 \%(2 / 9)$ in patients treated with anti-PD-1/PD-L1 monoclonal antibodies [8]. In another report, Lau et al. observed a RR of 50.0\% (3/6) and a median PFS of 4.8 months in patients undergoing ICB treatment [9]. Although these two works seem to suggest some benefit from treatment with immune checkpoint inhibitors in NSCLCs with EGFR Ex20ins mutations, it is authors' opinion that they are to be considered less reliable as compared with our results that have been obtained on a larger number of patients $(\mathrm{N}=15)$. By contrast, a study by Hastings et al. which took into account 28 patients with EGFR Ex20ins mutations treated with ICB reported a clinical outcome very similar to our study, with a RR of $10.7 \%(3 / 28)$, a PD of $64.3 \%(18 / 28)$, a median PFS of 1.9 months and a median OS of 5.5 months [10]. In addition, the same authors also associated the tumor mutation burden (TMB), which is an established marker of sensitivity to ICB, with a given EGFR mutation subtype, and found that EGFR Ex20ins mutations bear the lowest median levels of TMB (2.8 mutations $/ \mathrm{Mb}$ in 
19 evaluated patients) when compared with all other EGFR mutation subtypes, including common EGFR exon 19 deletion/L858R point mutations [10]. Accordingly, another study found low median levels of TMB (3.6 mutations/Mb) in 260 patients with EGFR Ex20ins mutations, with only $4 \%$ and $0.7 \%$ of these patients harboring intermediate-high (10 to 20 mutations $/ \mathrm{Mb}$ ) and high ( $>20$ mutations $/ \mathrm{Mb}$ ) TMB levels, respectively [11]. Certainly, the low levels TMB observed in NSCLCs with EGFR Ex20ins mutations are compatible with the predominant never/light smoking history reported by these patients and provide a strong molecular rationale in order to explain the poor activity of ICB treatment that we observed in this group of patients.

In the present study, we could not find any correlation between the sensitivity to treatment with ICB and a given Ex20ins variant (Figure 1C). In fact, the only patient who responded to immunotherapy was found to have the same Ex20ins variant as a patient who experienced progressive disease (V769_D770insAV), with both patients receiving an anti-PD-(L) 1 as monotherapy. Similarly, the only patient who had SD on immunotherapy possessed the same variant as two other patients who experienced progressive disease (H773_V774insNPH). This suggests that factors other than the type of Ex20ins variant could explain the reason why one patient responded to ICB treatment in our series. Recent studies have suggested that NSCLCs with uncommon EGFR mutations, including EGFR Ex20ins mutations, tend to be positive for CD8+ tumor infiltrating lymphocytes in approximately $50 \%$ to $60 \%$ of cases $[8,12]$. However, no correlation has been so far demonstrated between the levels of CD8+ tumor infiltrating lymphocytes and sensitivity to immunotherapy in NSCLC patients with uncommon EGFR mutations, which could be warranted in future correlative studies.

Whether ICB is active as first-line treatment for NSCLCs with EGFR Ex20ins mutations is a relevant question, given the fact that no standard therapeutic option exists for these patients besides chemotherapy. Importantly, we noted an inferior PFS and OS for patients who received ICB as first line treatment, although the difference did not reach statistical significance likely due to the small number of patients that were compared (6 versus 9 patients treated in the first and second line settings, respectively) (Figure 2B,E). These data suggest the absence of activity of first-line immunotherapy especially when used as single agent for patients with PD-L1 $\geq 50 \%$ (among the four PD-L1 $\geq 50 \%$ patients treated with pembrolizumab monotherapy in the first line setting $3 / 3$ underwent PD, while another patient underwent rapid deterioration of clinical conditions leading to death). At the present time, data on the use of single agent ICB in the first-line treatment of EGFR-mutant NSCLC have been published by Lisberg et al. and suggest a total lack of efficacy of pembrolizumab in the initial 'naïve' setting despite the presence of PD-L1 expression $\geq 1 \%$ [13]. Interestingly, the same study included 2 patients with EGFR Ex20ins mutations and PD-L1 $\geq 50 \%$, both of whom experienced PD as the best response.

Importantly, in our case series PD-L1 status was shown to have neither a predictive nor a prognostic role in relation to response to ICB treatment (Figure $2 \mathrm{C}, \mathrm{F}$ ). This absence of predictivity for PD-L1 is in line with other data that suggest the presence of an EGFR mutation may abrogate the predictive value of PD-L1 expression on response to ICB treatment $[10,14]$. The less relevant impact of PD-L1 expression in EGFR-mutant NSCLCs is also supported by the fact that lower levels of PD-L1 expression are generally present in case of EGFR mutation as compared with EGFR wild type tumors, as well as in comparison with NSCLCs with other driver mutations $[14,15]$. This is in line with the hypothesis that positive PD-L1 expression in patients with EGFR-mutant NSCLC more likely represents a constitutive activation of PD-L1 signal rather than a marker of adaptive immune response.

Overall, we also found that having received ICB treatment was associated with a worse survival from the time of diagnosis of advanced disease (Figure 3A,B), which was a factor found to be prognostic in the multivariate analysis. Apparently, this finding was not due to outperformance of patients in the group not receiving immunotherapy, as similar median survivals ranging between 24 and 26 months have been reported for patients with EGFR Ex20ins mutations not treated with immunotherapy $[16,17]$. By contrast, we can speculate 
that the poor survival of the immunotherapy group could be due to the fact that 4 out of the 15 patients treated with immunotherapy never received platinum-based chemotherapy and that these patients were less frequently administered platinum doublets (Tables 1 and S1). In addition, whether immunotherapy led to hyperprogressive disease in some cases that, in turn, could have had a negatively impact on the overall survival of patients treated with ICB, is a possibility that cannot be ruled out. Thus far, hyperprogressive disease has been already described in the setting of oncogene addicted NSCLC either at a preclinical or clinical level $[8,18,19]$.

By combining multiple patients from different institutions, we managed to include 30 patients with EGFR Ex20ins mutations in this study, which might be considered a relatively small sample size. Other possible limitations of this analysis are the retrospective nature and the inclusion of a population of patients heterogeneously treated with different immune checkpoint inhibitors either alone or in combination. In fact, while the majority of patients had received an anti-PD1 or anti-PD-L1 as monotherapy $(\mathrm{N}=12)$, few patients had received ICB combination regimens $(\mathrm{N}=3)$ either an anti-PD1 + anti-CTLA4 $(\mathrm{N}=1)$ or an anti-PD1 + platinum-based chemotherapy $(\mathrm{N}=2)$. Therefore, it is important that future studies will address the role of ICB given in combination regimens in NSCLCs with EGFR Ex20ins mutations, especially when given in combination with platinum-based chemotherapy.

\section{Conclusions}

In the present study, treatment with ICB was associated with a very poor clinical outcome in advanced NSCLCs with EGFR Ex20ins mutations, with only one response (6.7\%) and a dismal median PFS (2.0 months) and OS (5.3 months) observed. The worst treatment outcome was reported when ICB was used in the first line setting. In addition, patients treated with immunotherapy had a significantly worse OS from the time of diagnosis of advanced disease as compared with patients who never received ICB, which suggests that immunotherapy should be the least considered option in patients with EGFR Ex20ins mutation, to be reserved as a treatment option only after having administered standard platinum-based chemotherapy. On the other hand, more clinical data are needed in order to support the use of ICB in combination with platinum-based chemotherapy as first line treatment for these patients.

Supplementary Materials: The following are available online at https://www.mdpi.com/article/10 .3390/genes12050679/s1. Figure S1: Study flowchart, Table S1: Disposition of patients by the type of treatments received besides immunotherapy.

Author Contributions: Study conception and design: G.M. (Giulio Metro) and V.L.; Performed NGS analysis: V.L., S.B., M.S.R., F.R.T. and A.S. (Annamaria Siggillino); IHC analysis and provision of tumor tissue: G.B., M.M., M.T., E.P. and A.S. (Angelo Sidoni); Acquisition of clinical data: G.M. (Giulio Metro), C.M., A.G. (Antonella Giglietti), B.D.G., M.G. (Miriam Garaffa), F.M., V.M., M.G. (Marco Gunnellini), A.G. (Annalisa Guida) and M.S.; Analysis and interpretation of data: G.M. (Giulio Metro) and A.G. (Alessio Gili); Drafting of manuscript: G.M. (Giulio Metro); Critical revision and final approval of the manuscript: G.M. (Giulio Metro), S.B., G.B., M.M., A.G. (Alessio Gili), G.M. (Giovanni Marchetti), M.T., C.M., M.S.R., F.R.T., A.S. (Annamaria Siggillino), E.P., A.G. (Antonella Giglietti), B.D.G. (Bruna Di Girolamo), M.G. (Miriam Garaffa), F.M., V.M., M.G. (Marco Gunnellini), A.G. (Annalisa Guida), M.S., A.S. (Angelo Sidoni), F.R. and L.V. All authors have read and agreed to the published version of the manuscript.

Funding: This research received no external funding.

Institutional Review Board Statement: The study was conducted according to the guidelines of the Declaration of Helsinki and approved by the local Ethics Committee.

Informed Consent Statement: Informed consent was obtained from all individuals included in the study, whenever possible, taking into account the retrospective nature of the study. 
Data Availability Statement: The datasets generated and analyzed during the current study are available from the corresponding author on reasonable request.

Conflicts of Interest: The authors declare that they have no conflict of interest.

\section{References}

1. Planchard, D.; Popat, S.; Kerr, K.; Novello, S.; Smit, E.F.; Faivre-Finn, C.; Mok, T.S.; Reck, M.; Van Schil, P.E.; Hellmann, M.D.; et al. Metastatic non-small cell lung cancer: ESMO Clinical Practice Guidelines for diagnosis, treatment and follow-up. Ann. Oncol. 2018, 29, iv192-iv237. [CrossRef] [PubMed]

2. Bennati, C.; Paglialunga, L.; Ricciuti, B.; Metro, G.; Marcomigni, L.; Gili, A.; Crinò, L. Targeting EGFR and ALK in NSCLC: Current evidence and future perspective. Lung Cancer Manag. 2016, 5, 79-90. [CrossRef] [PubMed]

3. Zhang, T.; Wan, B.; Zhao, Y.; Li, C.; Liu, H.; Lv, T.; Zhan, P.; Song, Y. Treatment of uncommon EGFR mutations in non-small cell lung cancer: New evidence and treatment. Transl. Lung Cancer Res. 2019, 8, 302-316. [CrossRef] [PubMed]

4. Gainor, J.F.; Shaw, A.T.; Sequist, L.V.; Fu, X.; Azzoli, C.G.; Piotrowska, Z.; Huynh, T.G.; Zhao, L.; Fulton, L.; Schultz, K.R.; et al. EGFR Mutations and ALK Rearrangements Are Associated with Low Response Rates to PD-1 Pathway Blockade in Non-Small Cell Lung Cancer: A Retrospective Analysis. Clin. Cancer Res. 2016, 22, 4585-4593. [CrossRef] [PubMed]

5. Mazieres, J.; Drilon, A.; Lusque, A.; Mhanna, L.; Cortot, A.; Mezquita, L.; Thai, A.; Mascaux, C.; Couraud, S.; Veillon, R.; et al. Immune checkpoint inhibitors for patients with advanced lung cancer and oncogenic driver alterations: Results from the IMMUNOTARGET registry. Ann. Oncol. 2019, 30, 1321-1328. [CrossRef] [PubMed]

6. Yamada, T.; Hirai, S.; Katayama, Y.; Yoshimura, A.; Shiotsu, S.; Watanabe, S.; Kikuchi, T.; Hirose, K.; Kubota, Y.; Chihara, Y.; et al. Retrospective efficacy analysis of immune checkpoint inhibitors in patients with EGFR-mutated non-small cell lung cancer. Cancer Med. 2019, 8, 1521-1529. [CrossRef] [PubMed]

7. Schwartz, L.H.; Litière, S.; De Vries, E.; Ford, R.; Gwyther, S.; Mandrekar, S.; Shankar, L.; Bogaerts, J.; Chen, A.; Dancey, J.; et al. RECIST 1.1-Update and clarification: From the RECIST committee. Eur. J. Cancer 2016, 62, 132-137. [CrossRef] [PubMed]

8. Chen, K.; Pan, G.; Cheng, G.; Zhang, F.; Xu, Y.; Huang, Z.; Fan, Y. Immune microenvironment features and efficacy of PD-1/PD-L1 blockade in non-small cell lung cancer patients with EGFR or HER2 exon 20 insertions. Thorac. Cancer 2021, 12, $218-226$. [CrossRef] [PubMed]

9. Lau, S.C.; Fares, A.F.; Le, L.W.; Mackay, K.M.; Soberano, S.; Chan, S.W.; Smith, E.; Ryan, M.; Tsao, M.S.; Bradbury, P.A.; et al. Subtypes of EGFR- and HER2-Mutant Metastatic NSCLC Influence Response to Immune Checkpoint Inhibitors. Clin. Lung Cancer 2021, 1525. [CrossRef]

10. Hastings, K.; Yu, H.; Wei, W.; Sanchez-Vega, F.; DeVeaux, M.; Choi, J.; Rizvi, H.; Lisberg, A.; Truini, A.; Lydon, C.; et al. EGFR mutation subtypes and response to immune checkpoint blockade treatment in non-small-cell lung cancer. Ann. Oncol. 2019, 30, 1311-1320. [CrossRef] [PubMed]

11. Riess, J.W.; Gandara, D.R.; Frampton, G.M.; Madison, R.; Peled, N.; Bufill, J.A.; Dy, G.K.; Ou, S.-H.I.; Stephens, P.J.; McPherson, J.D.; et al. Diverse EGFR Exon 20 Insertions and Co-Occurring Molecular Alterations Identified by Comprehensive Genomic Profiling of NSCLC. J. Thorac. Oncol. 2018, 13, 1560-1568. [CrossRef] [PubMed]

12. Chen, K.; Cheng, G.; Zhang, F.; Zhu, G.; Xu, Y.; Yu, X.; Huang, Z.; Fan, Y. PD-L1 expression and T cells infiltration in patients with uncommon EGFR-mutant non-small cell lung cancer and the response to immunotherapy. Lung Cancer 2020, 142, 98-105. [CrossRef] [PubMed]

13. Lisberg, A.; Cummings, A.; Goldman, J.; Bornazyan, K.; Reese, N.; Wang, T.; Coluzzi, P.; Ledezma, B.; Mendenhall, M.; Hunt, J.; et al. A Phase II Study of Pembrolizumab in EGFR-Mutant, PD-L1+, Tyrosine Kinase Inhibitor Naïve Patients with Advanced NSCLC. J. Thorac. Oncol. 2018, 13, 1138-1145. [CrossRef]

14. Schoenfeld, A.J.; Rizvi, H.; Bandlamudi, C.; Sauter, J.L.; Travis, W.D.; Rekhtman, N.; Plodkowski, A.J.; Perez-Johnston, R.; Sawan, P.; Beras, A.; et al. Clinical and molecular correlates of PD-L1 expression in patients with lung adenocarcinomas. Ann. Oncol. 2020, 31, 599-608. [CrossRef] [PubMed]

15. Lee, S.E.; Kim, H.K.; Sung, M.; Han, J.; Choi, Y.-L. Association with PD-L1 Expression and Clinicopathological Features in 1000 Lung Cancers: A Large Single-Institution Study of Surgically Resected Lung Cancers with a High Prevalence of EGFR Mutation. Int. J. Mol. Sci. 2019, 20, 4794. [CrossRef] [PubMed]

16. Naidoo, J.; Sima, C.S.; Bs, K.R.; Busby, N.; Nafa, K.; Ladanyi, M.; Riely, G.J.; Kris, M.G.; Arcila, M.E.; Yu, H.A. Epidermal growth factor receptor exon 20 insertions in advanced lung adenocarcinomas: Clinical outcomes and response to erlotinib. Cancer 2015, 121, 3212-3220. [CrossRef]

17. Xu, C.-W.; Wang, W.-X.; Wang, D.; Wang, Q.-M.; Pu, X.-X.; Zhu, Y.-C.; Huang, J.-H.; Yu, Z.-Y.; Cui, Z.-L.; Chen, X.-H.; et al. Pemetrexed-based chemotherapy for non-small-cell lung cancer patients with EGFR exon 20 insertion mutation: A multicenter study. Transl. Lung Cancer Res. 2020, 9, 1853-1861. [CrossRef] [PubMed]

18. Lo Russo, G.; Moro, M.; Sommariva, M.; Cancila, V.; Boeri, M.; Centonze, G.; Ferro, S.; Ganzinelli, M.; Gasparini, P.; Huber, V.; et al. Antibody-Fc/FcR interaction on mac-rophages as a mechanism for hyperprogressive disease in non-small cell lung cancer subsequent to PD-1/PD-L1 blockade. Clin. Cancer Res. 2019, 25, 989-999. [CrossRef] [PubMed]

19. Huang, X.; Xia, L.; Lan, F.; Shao, Y.W.; Li, W.; Xia, Y. Treatment of Nivolumab Results in Hyperprogressive Disease in a Patient Harboring EGFR Exon 20 Insertion and MYC Amplification. J. Thorac. Oncol. 2019, 14, e189-e191. [CrossRef] [PubMed] 